\title{
REVISÃO ETNOBOTÂNICA DE TYPHA L. (TYPHACEAE) NO BRASIL
}

\author{
ETHNOBOTANICAL REVIEW OF TYPHA L. (TYPHACEAE) IN BRAZIL
}

\author{
Jordano Dorval Tavares de Carvalho ${ }^{1 *}$, Mabel Rocio Báez-Lizarazo1, \\ Mara Rejane Ritter ${ }^{1,2}$
}

\begin{abstract}
Resumo
Typha L. (Typhaceae) é um gênero de monocotiledôneas aquáticas amplamente distribuído no mundo, o qual tem sido utilizado desde a pré-história como importante fonte alimentícia para o ser humano. Apesar disso, pouco se sabe sobre os aspectos etnobotânicos desse gênero no Brasil. Assim, conduziu-se esta revisão bibliográfica, utilizando as plataformas Google Scholar, Scopuse Web of Science. O presente estudo sintetiza o conhecimento difuso dos usos etnobotânicos de Typha, passados e presentes, por comunidades brasileiras, assim como sua distribuição, identificando as particularidades desse uso quando comparadas a outras culturas. Dentre os 28 trabalhos encontrados, o artesanato foi o principal uso referido, seguido de sua utilização na alimentação. A utilização de Typha como planta medicinal foi raramente relatada e nenhuma pesquisa citou aspectos rituais e místicos, embora este uso seja referenciado em outros países. As espécies de Typha estão distribuídas em todo o Brasil, exceto na região Amazônica, dessa forma a maioria dos trabalhos encontrados foi realizada no litoral brasileiro, principalmente nas regiões Sudeste e Sul. Tais resultados refletem a relação desse grupo de plantas com as populações que coabitam os ambientes em que elas ocorrem, como as lagoas litorâneas e a restinga. Percebe-se, também, que os trabalhos se concentram em regiões historicamente mais estudadas do ponto de vista botânico, para as quais há mais registros de ocorrência.
\end{abstract}

Palavras-chave: artesanato; planta alimentícia; taboa; totora; Typha domingensis, Typha latifolia

\section{Abstract}

Typha L. (Typhaceae) is a genus of aquatic monocotyledons widely distributed, which

\footnotetext{
${ }^{1}$ Programa de Pós-Graduação em Botânica, Universidade Federal do Rio Grande do Sul, Avenida Bento Gonçalves, 9500, CEP 91501-970, Porto Alegre, Rio Grande do Sul, Brasil.

${ }^{2}$ Departamento de Botânica, Universidade Federal do Rio Grande do Sul, Avenida Bento Gonçalves, 9500, CEP 91501-970, Porto Alegre, Rio Grande do Sul, Brasil. * jordano.tavares@ufrgs.br
} 
has been used since prehistory as an important food source for humans. Despite this, little is known about the ethnobotanical aspects of this genus in Brazil. Thus, this literature review was conducted using Google Scholar, Scopus, and Web of Science platforms. This review summarizes the widespread knowledge of past and present ethnobotanical uses of Typha, by Brazilian communities as well as its distribution. In addition, we aims identifies particularities of this use when compared to other cultures. Among the 28 works found, handicraft was the main use mentioned, followed by use in food. The use of Typha as a medicinal plant was rarely reported and no studies mentioned ritual and mystical aspects, although this use is referenced to other countries. Typha species are distributed throughout Brazil, except in the Amazon region, thus most of the works found were carried out on the Brazilian coast, mainly in the Southeast and South regions. These results reflect the relationship of this genus with the populations that coexist in the environments in that they occur, such as coastal lagoons and restinga. It is also noticed that the works are concentrated in regions historically more studied from the botanical point of view, for which there are more records of occurrence.

Keywords: food plant; handicraft; taboa; totora; Typha domingensis, Typha latifolia.

\section{Introdução}

A importância biológica e cultural das espécies de Typha L. se evidencia nos mais de 280 substantivos atribuídos a essas plantas por 63 famílias de línguas na África, Ásia, Europa e América (AUSTIN, 2007). O conhecimento e o uso das espécies desse gênero por diversas populações humanas veem desde o paleolítico superior (REVEDIN et al., 2010). A primeira citação de Typha, na literatura científica, é atribuída a Dioscórides (70 d.C.) em

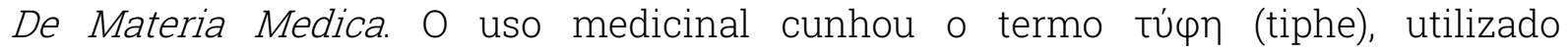
posteriormente por Lineu (1753) para designar o gênero, embora atualmente este uso seja pouco difundido. No Brasil, o nome comum mais frequente é taboa ou tabua(REITZ, 1984; KINUPP, 2007), além de tifa, encontrado em algumas referências e em dados de herbários. A relação histórica entre os grupos humanos e os táxons do gênero, por ser muito antiga, é difusa e diversa nas várias culturas do mundo, e inspirou estudos taxonômicos e etnobotânicos realizados em diferentes países (REVEDIN et al., 2010; ZHOU et al., 2018). Por exemplo, o uso alimentício remonta há mais de 30 mil anos (REVEDIN et al., 2010), evidenciando a importância cultural e histórica desse gênero, principalmente devido a sua abundância em áreas úmidas, sendo um importante recurso alimentício nesses ecossistemas.

Typha é um gênero monofilético da família Typhaceae (APG IV, 2016), que inclui 10 a 13 espécies e numerosos sinônimos, notoespécies e híbridos putativos (ZHOU et al., 2018). As espécies de Typha estão amplamente distribuídas, exceto na Antártida (KIM e CHOI, 2011; ZHOU et al., 2018), colonizando áreas úmidas, tais como lagos ou lagoas (Fig. 1A), pântanos de água doce ou salgada ou mesmo reservatórios contaminados por resíduos industriais.

São ervas rizomatosas que produzem inúmeros rametas (clones) com nomofilos lineares, de nervuras paralelas e bainha que pode ou não ser expandida em uma aurícula (SMITH, 1967; 1986). Na face ventral da bainha podem ocorrer "glândulas de mucilagem" escuras, cuja presença caracteriza algumas espécies e híbridos, como Typha domingensis Pers. (SMITH, 1986). A sinflorescência de Typha é subentendida por um longo pedúnculo e inclui até quatro ordens de ramificação onde as flores estão densamente dispostas 
(CARVALHO e MARIATH, 2019). As flores pistiladas estão localizadas na base e as estaminadas no ápice, podendo ou não haver uma lacuna entre as duas porções (Fig. 1B). As inúmeras flores pistiladas férteis apresentam um único pistilo e uma única semente, enquanto as estéreis (carpódios) tem estilete ou estigma reduzidos e um ovário expandido e suculento. As flores estaminadas são aperiantadas e possuem de um a oito estames, com anteras de deiscência rimosa e um prolongamento suculento no ápice. Curiosamente, segundo Müller-Doblies (1970), essas sinflorescências são as mais densamente arranjadas de todas as angiospermas, havendo contagem de 20 mil a mais de 700 mil frutos na porção pistilada de uma única inflorescência (GRACE e HARRISON, 1986). Os grãos de pólen são dispersos em tétrades ou mônades, sendo este um dos caracteres mais utilizados na delimitação das espécies e dos agrupamentos infragenéricos (KRONFELD, 1888; KIM e CHOI, 2011; ZHOU et al., 2018).

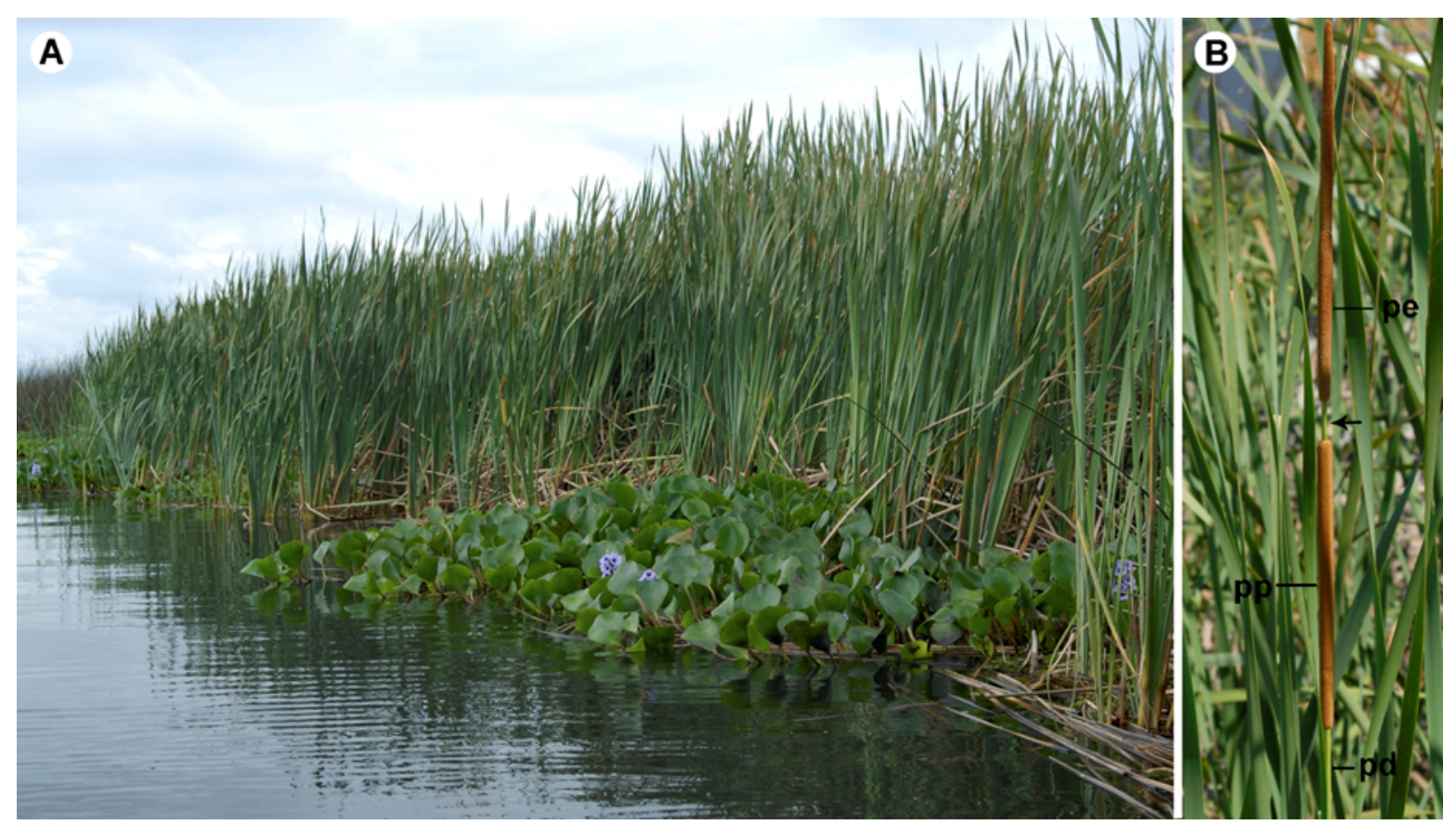

Figura 1. Habitat natural de Typha e detalhe da sinflorescência. A - Typha domingensis, na beira de um lago no município de Imbé - Rio Grande do Sul. B - Partes da sinflorescência de T. domingensis. pe: porção estaminada; pp: porção pistilada; pd: pedúnculo; seta: lacuna da sinflorescência. (Fotos de Jordano D. T. Carvalho).

No Brasil, atualmente, há três espécies segundo a Flora do Brasil 2020 (PAIVA et al., 2021): Typha angustifolia L., T. domingensis Pers. e T. latifolia L.. Nesse gênero é comum ocorrência de híbridos putativos (SMITH, 1986; KUEHN e WHITE, 1999; KIM e CHOI, 2011; CARVALHO, 2019), além da plasticidade de vários caracteres diagnósticos, o que gera dificuldades na identificação de espécies. Apesar disso, há estados de caráter que permitem sua identificação, por exemplo: T. latifolia é a única espécie com grãos de pólen em tétrade e apresenta flores pistiladas não subentendidas por bractéolas. Já a identificação de $T$. angustifolia e $T$. domingensis é mais sutil, pois ambas possuem bractéolas nas flores pistiladas e grãos de pólen em mônades, mas podem ser diferenciadas pela presença de glândulas escuras na face interna da bainha em $T$. domingensis. Outras características morfológicas da sinflorescência, como a lacuna entre as duas porções e a coloração da porção pistilada, são úteis na identificação de 
espécies e híbridos (notoespécies), estes podendo apresentar características compartilhadas com mais de uma espécie (CARVALHO e MARIATH, 2019).

Estudos apontam diferentes usos das espécies do gênero, como na alimentação de culturas tradicionais da América do Norte, os Apaches, por exemplo, e da América Central e do Sul, pelos Toltecas e tribos Chaquenhas, onde as inflorescências jovens e caules são utilizados como fonte de amido ou os abundantes grãos de pólen como fonte de proteína (LINSKENS e JORDE, 1997; ARENAS e SCARPA, 2003; KINUPP, 2007). Além disso, há relatos de uso artesanal, medicinal, tecnológico e ritual, por grupos humanos que coexistem nos ambientes habitados por essas plantas, como o uso das folhas, após secagem e manufatura, para a produção de artesanato ou para uso tecnológico, como vedação de barcos e casas e na produção de instrumentos para pesca (KINUPP, 2007). Tais espécies são consideradas plantas sagradas ou místicas para várias culturas no mundo, em comunidades originárias das Américas, África, Ásia e Europa (LINSKENS e JORDE, 1997; AUSTIN, 2007). Este uso reporta ao caso dos Astecas que utilizavam seus grãos de pólen para cobrir os rostos dos cativos destinados ao sacrifício humano, além de oferecerem os biscoitos produzidos com o pólen às divindades da guerra e aos reis. Os Toltecas (Tollin $=$ Typha, Juncus, teca $=$ existir, estar com) tem seu nome derivado do nome vernacular das tifas e outras plantas aquáticas comuns no território dessa civilização (AUSTIN, 2007). Porém, apesar desses registros, quase nenhuma informação existe sobre a utilização mística deste gênero para civilizações da América do Sul (AUSTIN, 2007).

No Brasil, com exceção de alguns levantamentos do uso de plantas nos quais as espécies de Typha são frequentemente listadas, não se tem uma visão mais específica de sua utilização nas diferentes regiões do país. Assim, nesta revisão, nós descrevemos e discutimos o atual conhecimento etnobotânico do gênero Typha no país, baseado na literatura, com ênfase nas espécies usadas e em suas categorias de uso com partes associadas no perfil das comunidades e localização dos estudos, bem como na distribuição dessas espécies.

\section{Material e Métodos}

Esta revisão foi baseada em artigos científicos, trabalhos de conclusão de curso, dissertações e teses obtidos por meio de buscas nas plataformas Google Scholar (https://scholar.google.com.br/), Scopus (https://www.scopus.com) e Web of Science (https://www.webofknowledge.com). As palavras-chave foram procuradas em inglês e português em diferentes combinações: Brasil, Etnobotânica, Typha, Typhaceae, uso, sem limite de data e separadas por vírgula.

Para a exclusão de trabalhos, primeiramente se analisou o título e o resumo das obras para que, posteriormente, os mesmos fossem acessados na íntegra. Excluiu-se os que se baseavam em outros trabalhos. Os resultados da busca foram considerados quando incluíam dados sobre qualquer categoria de uso das espécies de Typha por comunidades indígenas, quilombolas, de pescadores, rurais ou urbanas. Excluiu-se desse levantamento os usos biotecnológicos, sobretudo urbanos, como a utilização para fitorremediação de esgotos domésticos ou a extração de compostos ativos. Nos casos em que havia artigos e dissertações/teses/trabalhos de conclusão de curso com os mesmos autores e temas, foram selecionados os artigos. No entanto, se os artigos incluíssem somente uma parte do trabalho, as dissertações/teses e trabalhos de conclusão de curso também foram incluídos na nossa base de dados. 
É válido salientar que as categorias de uso citadas nos trabalhos foram: alimentício (AL), quando utilizadas para alimentação humana; artesanal (AR), quando utilizadas para a confecção de artesanato; medicinal (ME), quando utilizadas na medicina popular; tecnológica (TE), quando utilizadas na construção de casas, barcos ou na produção de ferramentas.

A lista das espécies com nomes válidos de Typha para o Brasil foi obtida na base de dados da Flora do Brasil 2020 (http://floradobrasil.jbrj.gov.br/). A partir disso, os dados de distribuição para exemplares depositados em herbários foram obtidos nas seguintes bases de informações: Herbário Rirtual Reflora (http://reflora.jbrj.gov.br/reflora/herbarioVirtual/), Global Biodiversity Information Facility (GBIF: http://www.gbif.org/) e SpeciesLink (www.splink.org.br). Cada um dos registros foi cuidadosamente conferido e quando um espécime tinha na etiqueta dados da localidade sem coordenadas, estes foram manualmente georreferenciados por meio do Google Earth (https://www.google.com/earth/) sobre The World Geodetic System (WGS84). Os dados de distribuição obtidos foram limpos usando o pacote CoordinateCleaner (ZIZKA et al., 2019) no software $R$, eliminando registros duplicados, coordenadas imprecisas, pontos no mar e capitais de cidades. Posteriormente, a distribuição resultante foi comparada com os dados de distribuição apresentados pela Flora do Brasil 2020 (2021) e, em casos de inconsistências, os registros foram novamente revisados. Os mapas de distribuição das espécies de Typha e seus usos relacionados com base na revisão de literatura foram criados usando ArcMap 10.5(ESRI, 2016).

\section{Resultados e Discussão}

Ao todo, apenas com as palavras-chave Brasil, Typha e uso, mil resultados da busca continham trabalhos relacionados a diferentes temas destacando-se os relacionados à fitorremediação e biotecnologia. Após a eliminação dos estudos com temas não etnobotânicos, obtivemos 28 trabalhos: 17 artigos científicos, um resumo expandido, 4 trabalhos de conclusão de curso, 4 dissertações, 1 tese e 1 livro. A espécie mais citada nesses trabalhos é Typha domingensis (16), seguida de T. angustifolia (4). Quatro trabalhos citavam apenas Typha sp. e um trabalho citou as duas espécies.

Foram encontrados registros etnobotânicos de Typha nos seguintes estados do Brasil: Bahia (BA), Espírito Santo (ES), Minas Gerais (MG), Mato Grosso (MT), Pernambuco (PE), Piauí (PI), Paraná (PR), Rio Grande do Sul (RS), Rio de Janeiro (RJ), Santa Catarina (SC), Sergipe (SE) e São Paulo (SP). As informações sobre os usos de Typha citados nesses trabalhos podem ser consultados na Tabela 1 e a distribuição dos mesmos na Figura 2.

Tabela 1. Espécie, categorias de uso, parte utilizada, comunidade/local para onde são reportados os usos etnobotânicos de táxons de Typha no Brasil e referências. AL (alimentício), AR (artesanal), ME (medicinal), TE (tecnológico).

\begin{tabular}{|c|c|c|c|c|}
\hline Espécie citada & $\begin{array}{c}\text { Categorias } \\
\text { de uso }\end{array}$ & $\begin{array}{c}\text { Parte } \\
\text { utilizada }\end{array}$ & Comunidade/Local & Referência \\
\hline \multirow[t]{3}{*}{ Typha angustifolia } & \multirow{3}{*}{$\mathrm{AL}$} & \multirow{3}{*}{$\begin{array}{l}\text { Não } \\
\text { informado }\end{array}$} & $\begin{array}{l}\text { Comunidades caiçaras/Ilha } \\
\text { do Cardoso-SP e Ilha de Santa } \\
\text { Catarina-SC }\end{array}$ & MIRANDA (2006) \\
\hline & & & $\begin{array}{l}\text { Comunidade rural/Viçosa- } \\
\text { MG }\end{array}$ & BARREIRA et al. (2015) \\
\hline & & & $\begin{array}{l}\text { Comunidades tradicionais } \\
\text { urbanas/Picos-PI }\end{array}$ & SANTOS et al. (2017) \\
\hline
\end{tabular}




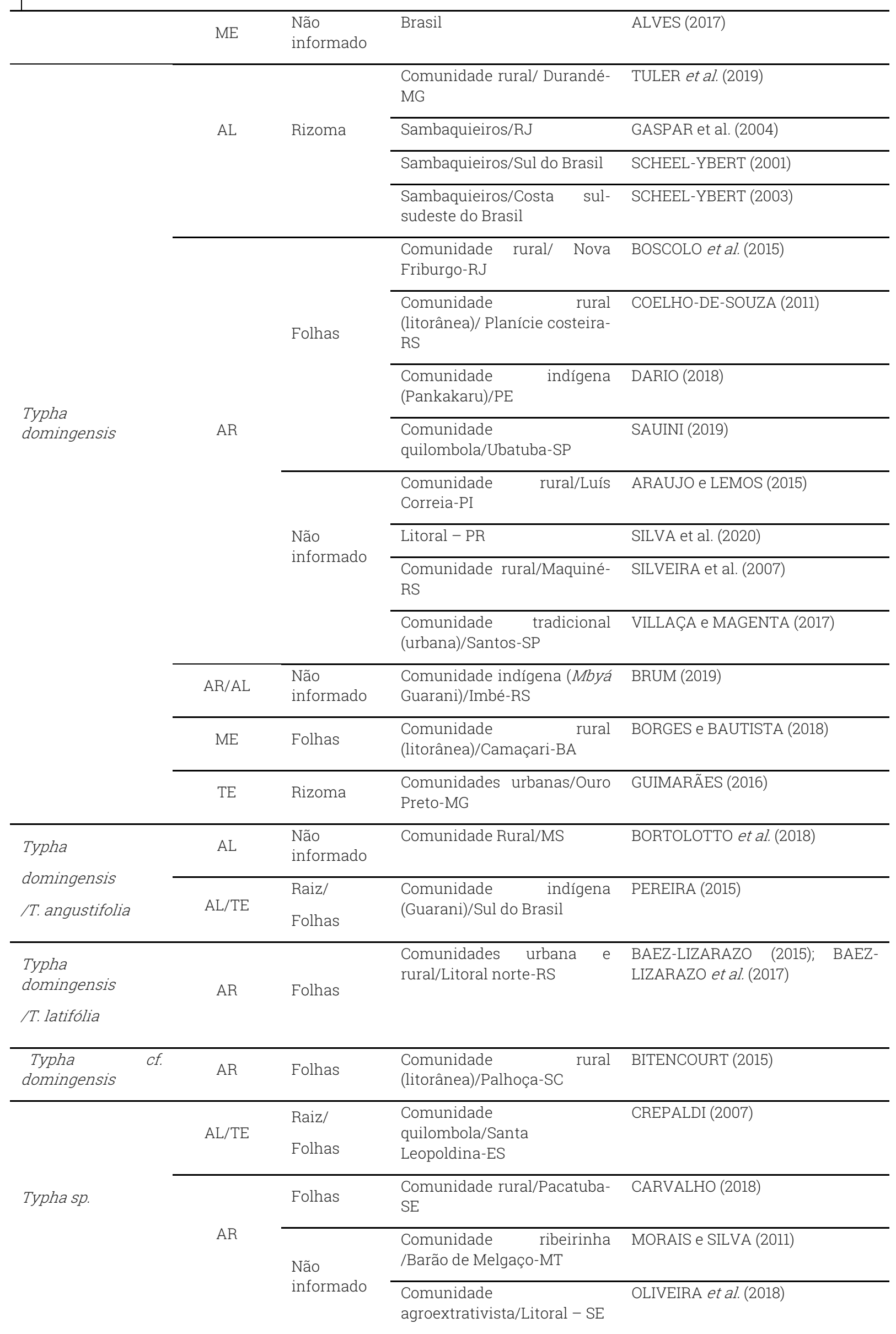


Foram encontrados 1.153 registros de exemplares de Typha depositados em herbários, dos quais 558 registros formaram a nossa base de dados final. Os táxons estão distribuídos quase que uniformemente no Brasil, exceto nos estados do Acre (AC), Amazonas (AM), Rondônia (RO) e Roraima (RR). Typha domingensis apresenta o maior número de registros (397) e a maior distribuição seguida por registros somente até gênero (73), T. angustifolia(53) e T. latifolia(33) (Fig. 2, A-D). Essas duas últimas espécies também apresentam distribuição descontinua no país que, junto aos registros de espécies determinadas até gênero, são um indicativo da falta de estudos taxonômicos e de outras áreas que permitam conhecer as espécies e as comunidades que as usam.

Para todos os taxóns a maior concentração de estudos etnobotânicos está localizada no litoral ou próximo do litoral Brasileiro, principalmente na região Sul e Sudeste do Brasil (Fig. 2), embora as espécies não sejam restritas a estes locais.

A. Typha angustifolia

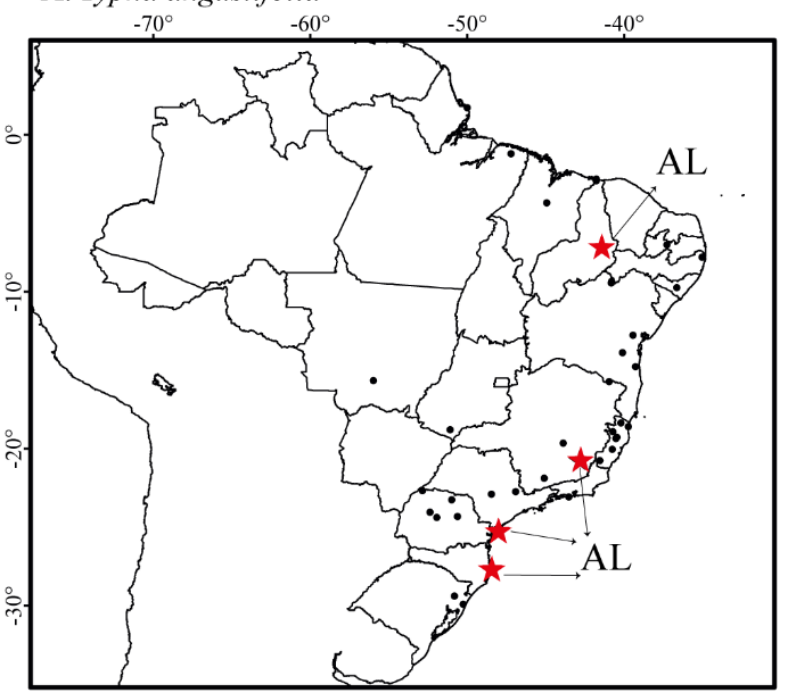

C. Typha latifolia

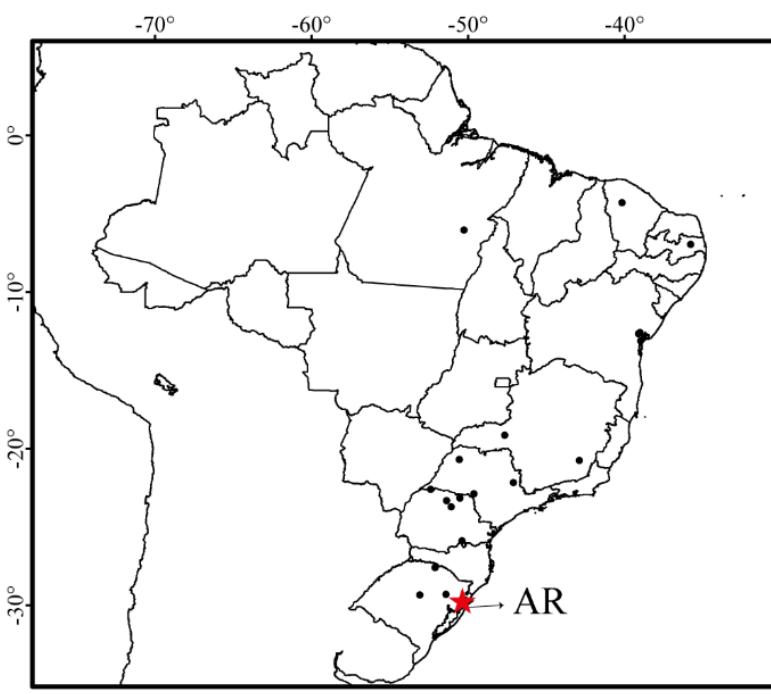

B. Typha domingensis

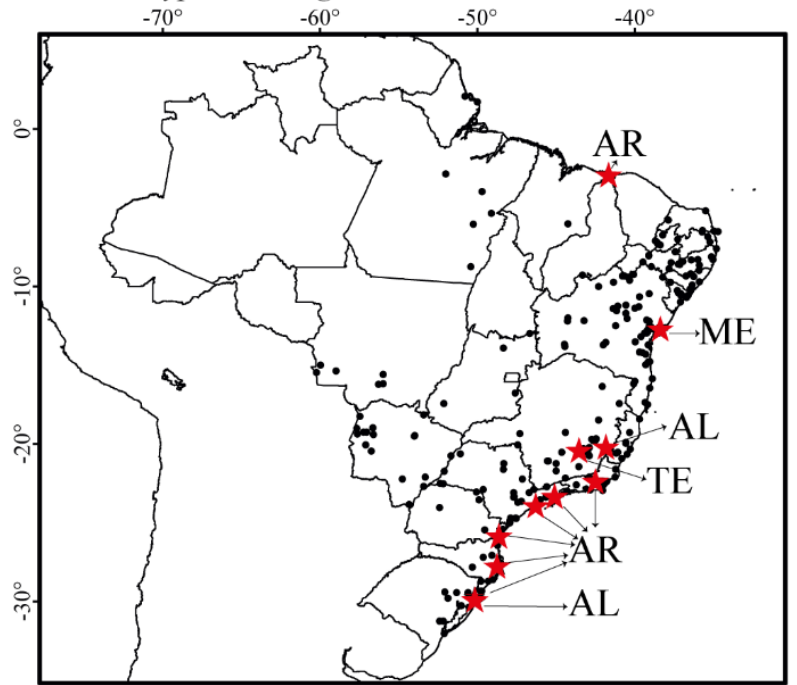

D. Typha sp.

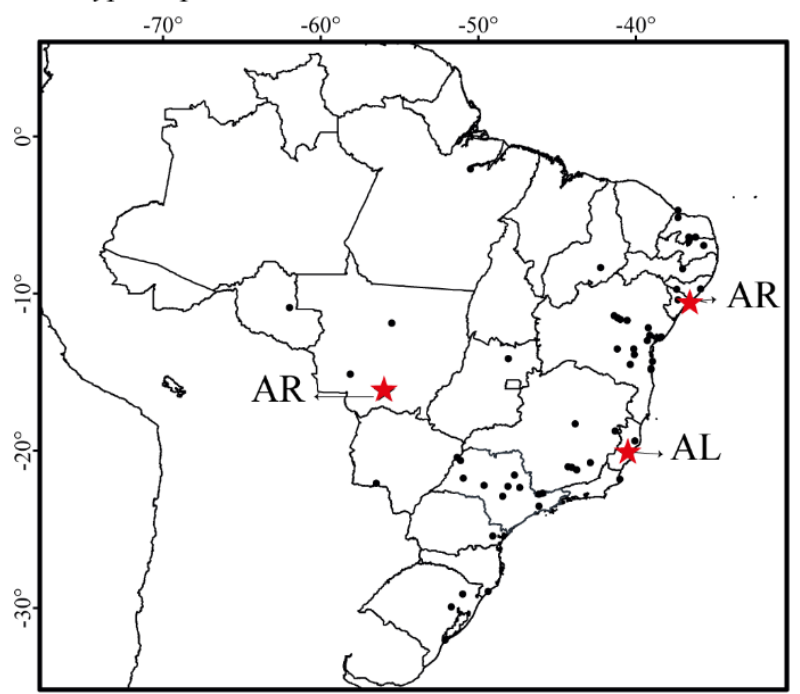

Figura 2. Distribuição geográfica e de usos dos taxóns de Typha no Brasil. (A-D) Distribuição das espécies de Typha (círculos) e os locais onde há registro de usos (estrelas). As letras nos mapas indicam os usos conhecidos para táxons de Typha: AL (alimentício), AR (artesanal), ME (medicinal), TE (tecnológico).

A categoria de uso com maior número de citações foi a artesanal, com 10 artigos científicos (SILVEIRA et al., 2007; MORAIS e SILVA, 2011; BOSCOLO et al., 2015; ARAUJO e LEMOS, 2015; BAEZ-LIZARAZO et al., 2017; SANTOS et al., 2017; VILLAÇA e MAGENTA, 
2017; DARIO, 2018; OLIVEIRA et al., 2018; SILVA et al., 2020), 2 trabalhos de conclusão de curso (BITENCOURT, 2015; BRUM, 2019), 2 dissertações (BAEZ-LIZARAZO, 2015; SAUINI, 2019), 1 tese (CARVALHO, 2018) e 1 livro (COELHO-DE-SOUZA, 2011). A maioria dos trabalhos teve como foco agricultores familiares e pescadores artesanais que produzem artesanato para uso próprio e para venda em comércio local. O uso artesanal tradicional foi citado para os povos indígenas Guaranis do Rio Grande do Sul e para o povo indígena Pankakarude Pernambuco. Apenas um trabalho citou a utilização por quilombolas. Além do uso artesanal, propriamente dito, em quatro casos foi referenciado o uso tecnológico, com a utilização das flores como isca para peixes e as folhas para a construção de casas (Tabela. 1).

A maioria dos artigos relatam apenas o produto final da manufatura, sem detalhes das etapas de elaboração. Em contrapartida, os trabalhos de conclusão de curso, as dissertações, a tese e o livro (COELHO-DE-SOUZA, 2011; BITENCOURT, 2015; BAEZLIZARAZO, 2015; PEREIRA, 2015; GUIMARÃES, 2016; CARVALHO, 2018; BRUM, 2019; SAUINI, 2019) além do produto final, trazem detalhes do processamento das fibras das folhas, a divisão de trabalho na comunidade (corte de matéria-prima, preparação, tecelagem e venda), além de aspectos econômicos, já que o artesanato produzido é a renda principal ou complementar dos membros dessas comunidades, ou por que são utilizados para o próprio benefício.

Há relatos de divisão do trabalho com os homens cortando as plantas e as mulheres produzindo as peças de artesanato, como em comunidades litorâneas do Rio Grande do Sul, onde as folhas de Typha domingensis e T. latifolia em conjunto com outras espécies e famílias botânicas (Juncaceae e Cyperaceae) são amplamente utilizadas (BAEZLIZARAZO, 2015; BAEZ-LIZARAZO et al., 2017). Já em outra comunidade do estado de Sergipe é relatado por CARVALHO et al. (2018) que as mulheres assumem todo o processo de manufatura do artesanato de Typha e o uso dessas plantas é considerado sustentável.

No Brasil são elaborados diversos produtos manufaturados incluindo cestas, esteiras para confecção de móveis, tapetes, cortinas, bolsas, bandejas, caixas, decorativos de mesa, chapéus, leques, dentre outros. Essa diversidade de utensílios também é observada em outros países nos quais se avaliou o uso artesanal de Typha (PARSAPAJOUH e GHAHREMANINEJAD, 2006), sendo relacionado às características específicas das folhas. Estas são longo-lineares, resistentes e maleáveis, de fácil manipulação e abundantes nas inúmeras rametas, além das espécies que formam grandes populações com rápida propagação vegetativa. Tais características também poderiam explicar o uso tecnológico das folhas de Typha, relatado em alguns trabalhos (MORAIS e SILVA, 2011; GUIMARÃES, 2016; SAUINI, 2019). Entre outras aplicações, as folhas podem ser usadas na vedação de barcos e casas. A produção de fibra é geralmente utilizada na construção de embarcações, já nas casas são usadas grandes quantidades de folhas agrupadas em feixes ou em combinação com barro e madeira, (GRACE e HARRISON, 1986).

Quase um terço dos trabalhos comentaram o uso alimentício de espécies de Typha: 5 artigos (SCHEEL-YBERT, 2001; GASPAR et al., 2004; BARREIRA et al., 2015; BORTOLOTTO et al., 2018; TULER et al., 2019), 1 resumo expandido (SCHEEL-YBERT, 2003), 2 dissertações de mestrado (MIRANDA, 2006; CREPALDI, 2007) e 2 trabalhos de conclusão de curso (PEREIRA, 2015; BRUM, 2019). O uso da planta por povos indígenas e quilombolas foi abordado num artigo e por comunidades rurais em quatro trabalhos (Tabela 1). O rizoma da planta com fim alimentício foi citado na maioria dos estudos como parte da lista das espécies apresentadas, sendo que apenas dois estudos detalharam o processo de preparação (BARREIRA et al., 2015; TULER et al., 2019). 
Três trabalhos recuperados em nossa revisão citam o uso do pólen como fonte alimentícia pelos sambaquieiros do Sudeste e Sul do país no Holoceno Superior (SCHEELYBERT, 2001; SCHEEL-YBERT, 2003; GASPAR et al., 2004). Tal dado evidencia a estreita relação dessas populações com o ambiente litorâneo e zonas de restinga ao longo do litoral brasileiro, cujos vestígios marcantes incluem os sambaquis e monólitos típicos desses povos. Porém, a relação dos sambaquieiros com as tifas que coabitavam essas regiões é, até então, hipotética, já que não foi avaliado o conteúdo taxonomicamente informativo dos grãos de pólen e amido, por exemplo, fazendo-se uma associação com as plantas recorrentes atualmente naquele ambiente. De qualquer forma, as plantas aquáticas e de restinga, típicas dos ambientes ocupados pelos sambaquieiros, certamente tiveram uma considerável importância na evolução desses povos, que podem as ter utilizado de diversas maneiras. Um cenário muito diferente, por exemplo, de outras populações pré-históricas estudadas na América do Sul, para as quais se relata o uso dos rizomas de Typha domingensis, comprovado pela análise dos grãos de amido em artefatos de moagem (MAZZ et al., 2014). Da mesma forma, evidências etnoarqueológicas (Revedin et al., 2010) apontam a utilização de Typha angustifolia e T. latifolia como plantas alimentícias por populações europeias ainda no Paleolítico. A metodologia utilizada pelos autores envolve não só a reconstrução do Paleoambiente e Paleoflora através de análise de grãos de pólen do período geológico, mas também comparam os grãos de amido oriundos da alimentação dessas populações, com o amido de espécies atuais.

Para populações mais recentes, PEREIRA (2015) e BRUM (2019) relatam o uso das tifas por comunidades Guarani para a alimentação e artesanato. Como não há uma indicação da parte da planta utilizada ou quais são os fatores culturais envolvidos nessa utilização, conjectura-se que nenhum desses trabalhos tenha ainda sido avaliado, uma vez que para Typha, aspectos mais profundos da etnobotânica envolvida na alimentação deste grupo. Em comunidades habitantes do litoral e restinga de Santa Catarina (SC) e São Paulo (SP), há relatos apenas da utilização alimentar deste gênero, sem abranger os aspectos culturais e históricos dessa utilização (MIRANDA, 2006). No entanto, estas comunidades ainda mantém uma relação forte com as plantas desses ambientes. O único trabalho a relatar o uso por quilombolas no Espírito Santo (ES) (CREPALDI, 2007) também não deixa claro qual parte utilizada ou com qual fim, embora o uso tecnológico combinado ao alimentar seja um indício da importância dessa planta para a comunidade.

Embora o rizoma tenha sido apontado como o único órgão utilizado como fonte de amido nos trabalhos analisados, Lorenzi e Kinupp (2014) citam também o palmito (gema apical do caule) de Typha como uma fonte de proteína e amido. Além disso é fato que a utilização alimentar dos grãos de pólen é de extrema importância histórica e cultural para comunidades atuais e ancestrais das Américas (LINSKENS e JORDE, 1997; ARENAS e SCARPA, 2003). Utilizado puro ou misturado a farinhas, os grãos de pólen das Typha podem ter sido tão importantes quanto o milho no início da evolução da cultura Maia (LINSKENS e JORDE, 1997).

O uso alimentício atual é bem documentado para comunidades indígenas do Gran Chaco e outras regiões da América do Sul (ARENAS e SCARPA, 2003) e América do Norte (DURHAM, 1951; KINUPP, 2007), as quais utilizam os grãos de pólen no preparo de biscoitos, pães e outros produtos. Essa tradição, embora não relatada em qualquer estudo desta pesquisa, é recuperada por Kinupp (2007) e Lorenzi e Kinupp (2014) ao abordarem amplamente a utilização atual dessas espécies como Plantas Alimentícias NãoConvencionais (PANC). Embora não cite a utilização dos grãos de pólen tradicionalmente 
no Brasil, seu consumo pode ter saído do âmbito tradicional dada a popularização de sua pesquisa. As PANC tomaram grande evidência na gastronomia do Brasil nas últimas décadas, num contexto em que a soberania alimentar, consumo de alimentos sem agrotóxicos, veganismo e outras questões se tornam cada vez mais importantes no contexto da pós-modernidade. Em contraponto, conforme nossas pesquisas, os usos de Typha angustifolia e $T$. domingensis como plantas alimentícias foram relatados em comunidades rurais, especialmente por idosos, que utilizavam o rizoma refogado, ou em molhos e caldos (BARREIRA et al., 2015; TULER et al., 2019). O consumo dessas plantas nessa faixa etária e em comunidades rurais indica o uso pouco frequente na tradição local.

O uso de Typha como planta medicinal foi citada apenas em dois artigos (Tabela 1), um deles fazendo referência ao uso importado da cultura chinesa, sem explicitar sua forma de uso (ALVES 2017). Numa comunidade litorânea da Bahia, as folhas Typha domingensis são utilizadas em infusão como anti-inflamatório (BORGES e BAUTISTA, 2018). Na medicina tradicional da Índia, China, Turquia e Paquistão e nas Américas, diferentes órgãos da planta são usados no tratamento de diversas doenças (KUMAR et al., 2013; SARDAR et al., 2014). Por exemplo, em comunidades da Turquia as folhas em infusão são utilizadas como diuréticas (SARDAR et al., 2014) e as flores pistiladas in natura para estancar hemorragias. As flores estaminadas e os grãos de pólen são usados para a cicatrização de feridas e hemostasia em diversas condições de hemorragia externa e interna, além de outros usos relacionados a condições inflamatórias, como febre e inchaço. O pólen de Typha também é utilizado na medicina tradicional Apache na América do Norte (LINSKENS e JORDE, 1997). Outras informações incluem o uso para alívio de dores abdominais e cólicas menstruais, dores do pós-parto e abcessos (SARDAR et al., 2014).

\section{Conclusões e novas perspectivas}

O uso artesanal foi o mais citado, seguido pelo alimentício. Poucas informações sobre as formas de uso medicinal foram encontradas e nenhum resultado sobre seu uso ritual/místico. A maior parte dos estudos têm sido realizados no litoral do Brasil ou em áreas próximas, indicando que nessas regiões a relação de Typha com comunidades ainda são mantidas, mas também pode ser um indicativo da concentração dos estudos nestas regiões, provavelmente pelo alto número de universidades e órgãos de pesquisa ali localizados.

Em grande parte dos estudos, os usos reportados integram listagens de levantamentos de dados etnobotânicos, sendo o uso artesanal o mais detalhado a nível cultural. Da mesma forma, seu uso alimentar necessita de uma investigação mais profunda nas diversas comunidades tradicionais do país, pois poucos detalhes sobre a parte consumida e forma de utilização são fornecidas nas investigações etnobotânicas. Adicionalmente, a utilização das espécies de Typha é relacionado e, ao mesmo tempo, poderia ser potencializado no Brasil devido à sua abundância e rápida reprodução vegetativa, levando a um uso sustentável. Estudos taxonômicos, de distribuição e etnobotânicos específicos para grupos de plantas auxiliam na construção de uma visão mais específica sobre a relação das comunidades humanas com essas espécies e com os seus habitats. 


\section{Agradecimentos}

Ao Conselho Nacional de Desenvolvimento Científico e Tecnológico (CNPq), pela bolsa de estudos concedida ao primeiro autor dessa pesquisa.

\section{Referências -}

ALVES, F. M. Orientação sobre o uso racional de plantas medicinais e fitoterápicos brasileiros na medicina tradicional chinesa. 2017. Monografia (Especialização em Gestão da Inovação em Fitomedicamentos). FIOCRUZ, RJ, Brasil. 69 p.

APG IV [Angiosperm Phylogeny Group IV]. An update of the Angiosperm Phylogeny Group classification for the orders and families of flowering plants: APG IV. Botanical Journal of the Linnean Society, v. 181, n.1, p. 1-20, 2016.

ARAUJO, J. L., LEMOS, J. R. Estudo etnobotânico sobre plantas medicinais na comunidade de Curral Velho, Luís Correia, Piauí, Brasil. Biotemas, v.28, p. 125-136, 2015.

ARENAS, P., SCARPA, G. F. The consumption of Typha domingensis Pers. (Typhaceae) pollen among the ethnic groups of the Gran Chaco, South America. Economic Botany, v. 57(2), n.181-188, 2003.

AUSTIN, D. F. Sacred Connections with Cat-tail (Typha, Typhaceae) - Dragons, waterserpents and reed-maces. Ethnobotany Research and Applications, v. 5, n. 273-303, 2007.

BAEZ-LIZARAZO, M. R. Estudo etnobotânico das plantas aquáticas vasculares para artesanato no litotal norte do Rio Grande do Sul-Brasil. 2015. Dissertação (Mestrado em Botânica). Universidade Federal do Rio Grande do Sul, RS, Brasil. 131 p.

BÁEZ-LiZARAZO, M. R., SANTORO, F. R., AlBUQUERQUE, U. P., RITTER, M. R. Aquatic vascular plants as handicraft: a case study in southern Brazil. Acta Botanica Brasilica, v.32, p. 88-98, 2017.

BARREIRA, T. F., FILHO, G. X. P., RODRIGUES, V. C. C., ANDRADE, F. M. C., SANTOS, R. H. S., PRIORE, S. E., PINHEIRO-SANT'ANA, H. M. Diversidade e equitabilidade de plantas alimentícias não convencionais na zona rural de Viçosa, Minas Gerais, Brasil. Revista Brasileira de Plantas Medicinais, v. 17, n. 964-974, 2015.

BITENCOURT, L. O artesanato de taboa (Typha cf. dominguensis Pers.) e junco (Androtrichum trigynum (Spreng.) H. Pfeiff.) na Guarda do Embaú, Palhoça, SC. 2015. Trabalho de Conclusão de Curso (Bacharelado em Ciências Biológicas). Universidade Federal de Santa Catarina, SC, Brasil. 51 p.

BORGES, K. N., BAUTISTA, H. Etnobotânica de plantas medicinais na comunidade de Cordoaria, litoral norte do estado da Bahia, Brasil. PLURAIS-Revista Multidisciplinar, v. 1, 2018. p. 153-174.

BORTOLOTTO, I. M., DAMASCENO-JUNIOR, G.A., POTT, A. Lista preliminar das plantas alimentícias nativas de Mato Grosso do Sul, Brasil. Iheringia Série Botânica, v. 73, 101-116, 2018.

BOSCOLO, O.H., VALENTE, L.R.R.M., SENNA-VALLE, L. Etnobotânica como ferramenta para identificação de indicações geográficas e marcas coletiva sem comunidade da região serrana do Rio de Janeiro. Gestão, Inovação e Tecnologias, v. 5, n.1, p. 1662-1673, 2015. 
BRUM, C.M. Levantamento de espécies vegetais presentes no Parque Estadual de Itapeva relevantes para a comunidade indígena da etnia Mbyá Guarani. 2019. Trabalho de Conclusão de Curso (Graduação em Ciências Biológicas). Universidade Federal do Rio Grande do Sul, RS, Brasil. 38 p.

CARVALHO, A.F.D. Mulheres artesãs: extrativismo da taboa (Typhaspp.) em Pacatuba/SE. 2018. Tese (Doutorado em Associação Plena em Desenvolvimento e Meio Ambiente). Universidade Federal de Sergipe, SE, Brasil. 230 p.

CARVALHO, J.D.T. Morfoanatomia e desenvolvimento de órgãos reprodutivos em espécies de Typha L. (Typhaceae). 2019. Dissertação (Mestrado em Botânica). Universidade Federal do Rio Grande do Sul, RS, Brasil. 97 p.

CARVALHO, J.D.T., MARIATH, J.E.A. Synflorescence morphology of species of Typha L. (Typhaceae): anatomical and ontogenetic bases for taxonomic applications. Acta Botanica Brasilica, v. 33, n. 4, n. 672-682, 2019.

COELHO-DE-SOUZA, G. Transformações no espaço rural. $1^{a}$ edição. Porto Alegre: Editora da Universidade Federal do Rio Grande do Sul, 2011. 121 p.

CREPALDI, M.O.S. Etnobotânica na Comunidade Quilombola Cachoeira do Retiro, Santa Leopoldina, Espírito Santo, Brasil. 2007. Dissertação (Mestrado em Botânica). Instituto de Pesquisas Jardim Botânico do Rio de Janeiro - Escola Nacional de Botânica Tropical, RJ, Brasil. 77 p.

DARIO, F.R. Uso de plantas da caatinga pelo povo indígena Pankararu no Estado de Pernambuco, Brasil. Revista Geotemas, v. 8, p. 60-76, 2018.

DURHAM, O. C. The pollen harvest. Economic Botany, v. 5, p. 211-254, 1951.

ESRI 2016. ArcGIS Desktop: Release 15. Redlands, CA: Environmental Systems Research Institute.

FLORA DO BRASIL 2020. Jardim Botânico do Rio de Janeiro. Disponível em: < http://floradobrasil.jbrj.gov.br/ >. Acesso em: 24 ago. 2020

GASPAR, M. D., TENÓRIO, M. C., BUARQUE, A., BARBOSA-GUIMARÃES, M., OLIVEIRA, J. C., SCHEEL-YBERT, R. Histórico e principais resultados do projeto de investigação: o aproveitamento ambiental das populações pré-históricas do Rio de Janeiro. Arquivos do Museu Nacional, v. 62, p. 103-129, 2004.

GRACE, J.B., HARRISON, S. The biology of Canadian weeds. 73. Typha latifolia L., Typha angustifolia L. and Typha x glauca Godr. Canadian Journal of Plant Science, v. 66, p. 361379, 1986.

GUIMARÃES, M.F.M. Plantas úteis em comunidades urbanas: a importância das espécies exóticas e do gênero na manutenção do conhecimento e uso dos recursos vegetais. 2016. Dissertação (Mestrado em Ecologia - Evolução e funcionamento de ecossistemas). Universidade Federal de Ouro Preto, MG, Brasil. 119 p.

KIM, C., CHOI, H-K. Molecular systematics and character evolutivon of Typha (Typhaceae) inferred from nuclear and plastid DNA sequence data. Taxon, v. 60, p. 1417 1428. 2011.

KINUPP, V. F. Plantas alimentícias não-convencionais da região metropolitana de Porto Alegre, RS. 2007. Tese (Doutorado em Fitotecnia - Horticultura). Universidade Federal do Rio Grande do Sul, RS, Brasil. 590 p. 
LORENZI, H., KINUPP, V. F. Plantas alimentícias não convencionais (PANC) no Brasil. Nova Odessa: Instituto Plantarum. 2014. 790 p.

KRONFELD, M. Monographie der Gattung Typha Tourn. (Typhinae Agdh., Typhaceae Schur-Engl.). Verhandlungen der Zoologisch-Botanischen Gesellschaft em Wien, v. 39, p. 89-192, 1888.

KUEHN, M. M., WHITE, B. N. Morphological analysis of genetically identified cattails Typha latifolia, Typha angustifolia and Typha x glauca. Canad. J. Bot., v. 77, p. 906-91, 1999.

KUMAR, K. S., KUMAR, D. S., TEJA, V. A., VENKATESWARLU, V., KUMAR, M. S., NADENDLA, R. R. A review on Typha angustata. International Journal Phytopharm, v. 4, p. 277-281, 2013.

LINNAEUS C. Species plantarum. 1753. Ed. I. Salvii. Estocolmo.

LINSKENS, H. F., JORDE, W. Pollen as food and medicine-a review. Economic Botany, v. 51, n. 1, p.78, 1997.

MAZZ, J. M. L., DABEZIES, J. M., CAPDEPONT, I. La gestión de recursos vegetales en las poblaciones prehistóricas de las tierras bajas del sureste del Uruguay: un abordaje multidisciplinar. Latin American Antiquity, v. 25, n. 3, p. 256-277, 2014.

MORAIS, F., SILVA, C. Etnoecologia de plantas nativas na comunidade de Estirão Comprido, Pantanal Matogrossense-Brasil. Revista de Ciências Agro-Ambientais, v. 9, p. 13-30, 2011.

MIRANDA, T. M. Etnobotânica de restinga em comunidades da ilha do Cardoso (SP) e da ilha de Santa Catarina (SC). 2006. Dissertação (Mestrado em Biologia Vegetal). Universidade Federal de Santa Catarina, SC, Brasil. 165 p.

MÜLLER-DOBLIES, D. Über die Verwandtschaft von Typha und Sparganium im Infloreszenzund Blütenbau. Botanische Jahrbucher fur Systematik Pflanzengesch. Pflanzengeogr., v. 89, n. 45, p. 1-562, 1970.

OLIVEIRA, D. M., SANTOS, L. A. S., GOMES, L. J. Uso da flora em assentamento agroextrativista do litoral de Sergipe, Brasil. Guaju, v. 4, p. 163-183, 2018.

PAIVA, G. C. P.; MATOS, A. M. M. V.; LOURENÇO, A. R.; BOVE, C. P. 2020. Typhaceae in Flora do Brasil 2020. Jardim Botânico do Rio de Janeiro. Disponível em: $<$ http://floradobrasil.jbrj.gov.br/reflora/floradobrasil/FB594210>. Acesso em: 15 jun. 2021

PARSAPAJOUH, S., GHAHREMANINEJAD, F. Ethnobotanical use of Typha domingensis Pers. (Typhaceae) in an arid zone: Sistan, Iran. Zonas Áridas, v. 8, p. 7-17. 2006.

PEREIRA, G. D. S. Utilização das plantas pelos Guarani. 2015. Trabalho de Conclusão de Curso (Bacharelado em Ciências Biológicas). Universidade do Extremo Sul Catarinense, SC, Brasil. 66 p.

REITZ, R. Tifáceas in: Reitz, R. (ed.). Flora Ilustrada Catarinense. 1984. Itajaí, Santa Catarina, Brasil, 14p.

REVEDIN, A., ARANGUREN, B., BECATTINI, R., LONGO, L., et al. Thirty thousand-year-old evidence of plant food processing. Proceedings of the National Academy of Sciences, $\mathrm{V}$. 107, n. 44, p. 18815-18819, 2010.

SANTOS, S. B. S., OLIVEIRA, Y. R., SILVA, P. H., ABREU, M. C. Percepções dos moradores acerca de Typha angustifolia subsp. domingensis (Pers.) Rohrb.(Typhaceae Juss.) em 
áreas alagadiças de Picos-PI, Nordeste do Brasil. Research, Society and Development v. 4, n. 3, p. 184-198, 2017.

SARDAR, A. A., KHAN, Z. U. D., PERVEEN, A., FARID, S., KHAN, I. U. In vitro antioxidant potential and free radical scavenging activity of various extracts of pollen of Typha domigensis Pers. Pakistan Journal of Pharmaceutical Sciences, v. 27, n. 2, p. 279-284, 2014.

SAUINI, T. Levantamento etnobotânico participativo entre os moradores do Quilombo do Cambury, Ubatuba, SP, Brasil. 2019. Dissertação (Mestrado em Biologia Química). Universidade Federal de São Paulo, SP, Brasil. 151 p.

SCHEEL-YBERT, R. Man and vegetation in southeastern Brazil during the late Holocene. Journal of Archaeological Science, v. 28, p. 471-480, 2001.

SCHEEL-YBERT, R. Relações dos habitantes de sambaquis com o meio ambiente: evidências de manejo de vegetais na costa sul-sudeste do Brasil durante o Holoceno Superior. 2003. In Atas do IX Congresso da Associação Brasileira de Estudos do Quaternário e II Congresso do Quaternário de Países de Línguas Ibéricas.

SILVA, L. E. D., AMARAL, W., SILVA, M., OLIVEIRA, A. L. D. Conservation of genetic resources: a study with medicinal plants on the coast of Paraná - Brazil. Ambiente \& Sociedade, v. 23, 2020. 20 p.

SILVEIRA, T. C. L., COELHO-DE-SOUZA, G., RODRIGUES, G. G. Crescimento, produção primária e regeneração de Typha domingensis Pers.: elementos para avaliação do uso sustentável da espécie. Revista Brasileira de Biociências: Brazilian Journal of Biosciences, v. 5, p. 678-680, 2007.

SMITH, S. G. Experimental and natural hybrids in North America. Typha (Typhaceae). Amer. Midi. Naturalist, v. 78, p. 257-287, 1967.

SMITH, S. G. The cattails (Typha): interspecific ecological differences and problems of identification. Lake and Reservoir Management, v. 2, n. 1, p. 357-362, 1986.

TULER, A. C., PEIXOTO, A. L., SILVA, N. C. B. D. Plantas alimentícias não convencionais (PANC) na comunidade rural de São José da Figueira, Durandé, Minas Gerais, Brasil. Rodriguésia, v. 70, e01142018, 2019. 12 p.

VILLAÇA, I. M., MAGENTA, M. A. G. Plantas utilizadas pela população de Monte Cabrão, Santos, SP, Brasil. Anais do Encontro Nacional de Pós Graduação, v. 1, n. 1, p. 286-290, 2017.

ZHOU, B., TU, T., KONG, F., WEN, J., XU, X. Revised phylogeny and historical biogeography of the cosmopolitan aquatic plant genus Typha(Typhaceae). Scientific reports, v.8, n. 1, p. $1-7,2018$.

ZIZKA, A., SILVESTRO, D., ANDERMANN, T., AZEVEDO, J., DUARTE RITTER, C., EDLER, D. SVANTESSON, S. CoordinateCleaner: Standardized cleaning of occurrence records from biological collection databases. Methods in Ecology and Evolution, v. 10, n. 5, p. 744-751, 2019. 